\title{
TRATAMIENTO DEL SUFRIMIENTO FETAL INTRAPARTO CON ORCIPRENALINA
}

TRABAJO PRESENTADO AL $X$ CONGRESO COLOMBIANO DE OBSTETRICIA $Y$ GINECOLOGIA.

5 al 8 de Diciembre de 1973 - Cali, Colombia.

UNIVERSIDAD DE CARTAGENA - FACULTAD DE MEDICINA - DEPARTAMENTO DE OBSTETRICIA

Y GINECOLOGIA.

Dr. Antonio Soto Yancés*

Dr. Domingo Palencia Ortega**

Dr. Jorge Milanés P.

Dr. Aníbal Perna M.****

\section{I - Introducción}

Uno de los principales objetivos actuales del obstetra es el de obtener un recién nacido en óptimo estado de vitalidad, dependiendo esta condición en gran medida de la agresión a que haya sido sometido el feto durante el trabajo de parto, es decir de la presencia o ausencia de sufrimiento fetal agudo. De acuerdo a la Escuela de Montevideo, el factor principal que causa dicho estado son las contracciones uterinas anormalmente elevadas, las cuales reducen los intercambios materno-fetales y llegan a producir trastornos en la homeostasis fetal. Se manifiestan externamente por modificaciones de la Frecuencia Cardíaca Fetal (F.C.F.), que pueden ser detectadas fácilmente por métodos clínicos.

Según investigaciones realizadas en la citada escuela, se sabe que generalmente, cuando se registran signos de sufrimiento fetal (taquicardia, Dips tipo II o bradicardia) durante el trabajo de parto, nacen niños deprimidos y con un índice de Apgar bajo ( 1 a 6).

Basándonos en la fisiopatología del surfimiento fetal agudo descrita por la ya mencionada Escuela de Montevideo y en los métodos clínicos propuestos por la misma, hemos relaizado el presente trabajo, utilizando la acción útero-inhibidora de la Orciprenalina*, la cual al producir inhibición de la contractilidad uterina, aumenta el flujo sanguíneo a través de la placenta y por ende el intercam-

* Profesor Asistente Depto. Obst. Ginec. Universidad de Cartagena. Obstetra Hospitalario ICSS.

** Obstetra Adscrito ICSS.

*** Profesor Emérito Depto. Obst. Ginec. Universidad de Cartagena.

$* * * *$ Profesor Jefe Depto. Obst. Ginec. Universidad de Cartagena. Obstetra Hospitalario ICSS.

* ALUPENT - C. H. Boehringer Sohn Ingelheim am Rheim, Alemania. 
bio metabólico materno-fetal, mejorando progresivamente el estado del feto in-útero.

\section{II - Material y Métodos}

El presente trabajo se realizó en la Clínica Universitaria Rafael Calvo C. Departamento de Obstetricia y Ginecología de la Universidad de Cartagena y en la Clínica del ICSS. de Cartagena, sobre 30 pacientes con embarazo a término en trabajo de parto espontáneo 0 inducido, a quienes se les diagnosticó la presencia de un sufrimiento fetal agudo por métodos clínicos, basados en los modelos básicos de la F.C.F. a saber:
A) Taquicardia.
B) Taquicardia y/o Dips Tipo II, y
C) Bradicardia permanente.

En todos los casos la Orciprenalina (Alupent) se utilizó por vía intravenosa continua, mediante la infusión de $500 \mathrm{ml}$. de Dextrosa al $5 \%$ en A. D. con 5 mgs. ( 10 ampollas) de Orciprenalina, a una velocidad de goteo variable entre 20 y 40 gotas por minuto (10 a 20 microgramos por minuto), de acuerdo a la evolución de cada caso y al efecto sobre las constantes materno-fetales. Estos parámetros fueron registrados clínicamente cada 10 minutos y la forma de terminar el parto (vaginal o cesárea) fué decidida según la evolución de cada caso en particular, por lo cual el tiempo de tratamiento fué diferente.

Sin excepción la infusión de Orciprenalina fue suspendida en el momento inmediatamente anterior al nacimiento y se reemplazó por una solución de 10 Unidades de Oxitocina en $500 \mathrm{ml}$. de Dextrosa. Los recién nacidos fueron clasificados en dos grupos, de acuerdo al Indice de Apgar registrado 1 y 5 minutos después del parto, a saber: Deprimidos (Apgar 1 a )6 y Vigorosos (Apgar 7 a 10).

\section{III - Resultados}

Los datos obtenidos fueron tabulados y procesados estadísticamente con los siguientes resultados:

A) Edad: La distribución de los 30 casos de acuerdo a grupos de edades se ilustra en el Cuadro 1.

\section{CUADRO № 1}

\begin{tabular}{lcc}
\hline Edad & No Casos & $\%$ \\
\hline $15-20$ & 2 & $27 \%$ \\
$20-25$ & 12 & $40 \%$ \\
$25-30$ & 6 & $20 \%$ \\
$30-35$ & 3 & $10 \%$ \\
$35-40$ & 1 & $3 \%$ \\
\hline
\end{tabular}

Se observa en nuestra casuística que la mayoría $(87 \%)$ de las pacientes tratadas son menores de 30 años y que solo un caso ( $3 \%$ ) pasó de los 35 años. Se trataba justamente de una primigesta añosa, a la cual se practicó cesárea y a quien se había diagnosticado el sufrimiento fetal mediante la prueba de tolerancia a las contracciones uterinas inducidas.

B) Paridad: Según la paridad la distribución es la siguiente:

\section{CUADRO N: 2}

\begin{tabular}{ccr}
\hline Paridad & No Casos & $\%$ \\
\hline 0 & 21 & $70 \%$ \\
1 & 6 & $20 \%$ \\
2 & 2 & $7 \%$ \\
3 & 1 & $3 \%$ \\
\hline
\end{tabular}

Se nota en el cuadro anterior que el $70 \%$ de las pacientes estudiadas eran nulíparas y el $20 \%$ primíparas lo que explica en principio la mayo- 
ría de pacientes jóvenes tratadas y en cierta forma la mayor incidencia de sufrimiento fetal en las nulíparas.

C) Relación entre el índice de Apgar al $1 \%$ y $5^{\circ}$ minuto y las modificaciones de la frecuencia cardíaca fetal.

Observamos en el Cuadro № 3 que en el grupo de pacientes que solo presentó taquicardia (4) como signo de sufrimiento fetal, la administración de la droga permitió la recuperación del feto en todos los casos y los cuatro recién nacidos registraron un Indice de Apgar entre 7 y 10 (Grupo Vigoroso) tanto al 1\% como al 50 minuto.

En el grupo de pacientes con taquicardia asociada a Dips Tipo II se obtuvieron dos (2) niños deprimidos

\section{CUADRO N 3}

\begin{tabular}{lcccc}
\hline F. C. F. & Apgar & ler. & Min. & \multicolumn{2}{c}{ Apgar } & $\mathbf{5}^{\mathbf{9}}$ & Min. \\
\hline TAQUICARDIA & $\mathbf{1}-\mathbf{6}$ & $\mathbf{7}-\mathbf{1 0}$ & $\mathbf{1}-\mathbf{6}$ & $\mathbf{7}-\mathbf{1 0}$ \\
TAQUICARDIA & - & 4 & -1 & 4 \\
DIPS TIPO II & 2 & 17 & 2 & 18 \\
BRADICARDIA & 4 & 3 & 3 & 5 \\
TOTALES & 6 & 24 & 27 \\
\hline
\end{tabular}

(Apgar 1 a 6) al ler. minuto, uno de los cuales siguió deprimido al $5^{\circ} \mathrm{mi}-$ nuto, mientras que el otro se recuperó satisfactoriamente y al $5^{\circ}$ minuto presentó Apgar de 9.

El tercer grupo formado por siete (7) pacientes con sufrimiento fetal grave caracterizado por bradicardia permanente, se obtuvieron cuatro (4) niños deprimidos y tres (3) vigorosos al ler. minuto, mientras que al $5 \circ$ minuto permanecían deprimidos dos (2) recién nacidos, habiéndose recuperado los otros dos (2). Vale aclarar que los dos niños que siguieron deprimidos al 5 : minuto, en ambos se encontró doble vuelta de cordón ceñida al cuello, lo que innegablemente dificultó la recuperación intrauterina de los mismos.

D) Relación entre el tiempo de tratamiento y el índice de Apgar al 1? y $5 \circ$ minutos.

\section{CUADRO N: 4}

\begin{tabular}{|c|c|c|c|}
\hline $\begin{array}{l}\text { Tiempo de adminis- } \\
\text { tración en minutos }\end{array}$ & $\begin{array}{l}\text { Apgar } \\
1-6\end{array}$ & $\begin{array}{l}\text { 1er. Min. } \\
7-10\end{array}$ & Total \\
\hline Menos de 20 & 2 & 0 & 2 \\
\hline $20-30$ & 1 & 2 & 3 \\
\hline $30-40$ & 1 & 7 & 8 \\
\hline $40-50$ & 0 & 11 & 11 \\
\hline $50-60$ & 0 & 4 & 4 \\
\hline Más de 60 & 2 & 0 & 2 \\
\hline TOTALES & 6 & 24 & 30 \\
\hline
\end{tabular}

Como podemos observar en el Cuadro $\mathrm{N}: 4$, en 2 de los recién nacidos deprimidos al ler. minuto el fármaco se administró durante un período menor de 20 minutos, contados entre el momento de la aplicación y la producción del parto, ambos casos por vía vaginal. En otros dos niños deprimidos la droga se administró por un lapso mayor de sesenta (60) minutos y ambos casos se obtuvieron por cesárea. Los dos (2) restantes correspondieron a pacientes en los 
cuales se aplicó entre los 20 y los 40 minutos respectivamente. Los veinticuatro (24) niños vigorosos ( $80 \%$ ) al ler. minuto, correspondieron a pacientes a los cuales se administró el fármaco opr un lapso comprendido entre los 20 y los 60 minutos, mientras que no se obtuvo ningún feto vigoroso cuando el tiempo de administración fue inferior a los $20 \mathrm{mi}$ nutos o superior a los 60 .

\section{CUADRO № 5}

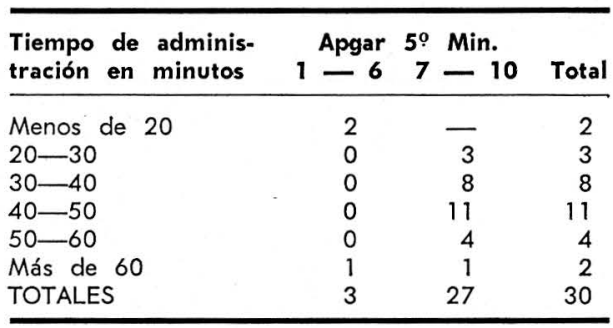

En el Cuadro № 5 observamos que de los 3 recién nacidos $(10 \%)$ que continuaron deprimidos al 5 \% minuto, dos (2) de ellos correspondieron a las pacientes a las cuales se administró la droga por un período menor de 20 minutos y cuyo parto fue por vía vaginal, mientras que el otro recién nacido fue obtenido por cesárea de una paciente que recibió la Orciprenalina, por más de 60 minutos. Los recién nacidos vigorosos al 5 ? minuto, 27 (90\%) correspondieron a pacientes a las cuales se administró la infusión con Orciprenalina por un período mayor de 20 minutos, estando la mayor incidencia entre los 30 y los 60 minutos.

\section{E) Relación entre el tipo de parto y el índice de Apgar.}

De las treinta (30) pacientes estudiadas, en $12(40 \%)$ se decidió el parto por vía vaginal y en las 18 restantes $(60 \%)$ se decidió practicar cesárea. La forma de finalizar el parto fue decidida de acuerdo a la evolución de cada caso en particular y teniendo en cuenta las normas del Departamento de Obstetricia y Ginecología de la Universidad de Cartagena.

\section{CUADRO N: 6}

\begin{tabular}{lcccccc}
\hline & Apgar & 1er. & Min. & Apgar & 5c & Min. \\
Tipo Parto & $\mathbf{1}-\mathbf{6}$ & $\mathbf{7}-\mathbf{1 0}$ & $\mathbf{1}-\mathbf{6}$ & $\mathbf{7}-\mathbf{1 0}$ & Total \\
\hline VAGINAL & 4 & 8 & 2 & 10 & 12 \\
CESAREA & 2 & 16 & 1 & 17 & 18 \\
TOTAL & 6 & 24 & 3 & 27 & 30 \\
\hline
\end{tabular}

El Cuadro № 6, ilustra los resultados obtenidos relacionando el tipo de parto con el puntaje de Apgar al 1 y 5 minutos respectivamente. Se observa que por vía vaginal cuatro (4) niños (13\%) estaban deprimidos al 1er. minuto, dos (2) de los cuales siguieron deprimidos al $5^{\circ} \mathrm{mi}-$ nuto, mientras que los otros dos (2) se recuperaron satisfactoriamente e ingresaron al grupo de los vigorosos $(33 \%)$. Los dos (2) niños que per- manecieron deprimidos correspondieron a los que presentaban doble vuelta de cordón al cuello. De los nacidos por cesárea, dos casos $(7 \%)$ estaban deprimidos al ler. minuto y $16(54 \%)$ vigorosos, mientras que al 5 ' minuto solo uno permaneció deprimido y el otro se recuperó e ingresó al grupo de los vigorosos $57 \%$.

Al hacer el análisis estadístico no se encontró diferencia significativa 
entre ambos grupos. Sinembargo, clínicamente el momento y la forma de decidir el parto (vaginal o cesárea) inciden grandemente en el estado de vitalidad fetal al nacimiento.

\section{IV - Efecto sobre las constantes materno-fetales. Frecuencia cardíaca materna}

La administración de Orciprenalina produjo en todos los casos marcada taquicardia materna, cuyo efecto apareció a los pocos minutos de iniciada la infusión y en especial cuando la dosis sobrepasó los 20 microgramos por minuto. En algunos casos (4) las pacientes se quejaron de palpitaciones cardíacas, las cuales desaparecieron al disminuir la velocidad de infusión.

\section{Tensión arterial materna}

En 14 casos se observó una baja muy pequeña de la tensión arterial materna, sin que en ningún momento comprometiera el estado general del paciente. Pudimos observar que en 11 de estos casos el descenso de la presión diastólica fue mayor que el de la sistólica, lo que produjo obviamente un ligero aumento de la presión diferencial. En todo caso, la acción de la droga sobre la tensión arterial materna fue de poca importancia y solo se presentó cuando la dosis fue mayor de 20 microgramos por minuto.

\section{Acción sobre la contractilidad uterina}

En todos los casos se observó inhibición de la contractilidad uterina y la intensidad dependió en cada caso de la dosis empleada. Su aparición empezó a notarse aproximadamente a los tres (3) minutos de iniciada la infusión. Observamos que a la dosis de 20 mcirogramos por minuto la in- hibición de la contractilidad uterina fue total en cada uno de los casos tratados, se hizo manifiesta aproximadamente a los 15 minutos del tratamiento.

\section{Efecto sobre la frecuencia cardíaca fetal}

Al igual que en la madre, la Orciprenalina causó en el feto aumento de la frecuencia cardíaca; este efecto fue mucho menor y de desarrollo progresivamente lento con relación a la F.C.M. Aproximadamente a los 15 minutos de tratamiento observamos en todos los casos una estabilización en la F.C.F., cuyas cifras sufrían poca modificación pese a seguirse la infusión.

En los casos del grupo A, en los cuales se administró la droga solamente por la presencia de Taquicardia fetal, se observó un aumento de la F.C.F. en valores máximos de 180 latidos por minuto y todos los fetos del grupo tuvieron al nacer un Puntaje de Apgar vigoroso (entre 7 y 10), lo que comprueba clínicamente que la taquicardia fetal producida por la Orciprenalina no es signo, ni agrava el sufrimiento fetal.

En los casos del grupo B (Taquicardia y/o Dips Tipo II) se observó aumento de la F.C.F. a valores máximos de 170 latidos por minuto con desaparición de los Dips Tipo II e inhibición de la contractilidad uterina aproximadamente a los 15 minutos de iniciada la infusión y a una dosis media entre 10 y 20 microgramos por minuto.

Los casos del grupo C (Bradicardia permanente) también manifestaron aumento en la F.C.F. en valores que oscilaron entre los 100 y 140 latidos por minuto, siendo los recién nacidos de este grupo los que ofre- 
cieron mayor dificultad a la recuperación intrauterina.

\section{$\checkmark$ - Evolución de los recién nacidos deprimidos}

Debemos informar que los tres recién nacidos que continuaron deprimidos al 5 ? minuto, fueron seguidos, tratados y evolucionados por personal especializado con los siguientes resultados:

Dos niños presentaron al $10^{\circ}$ minuto un Indice de Apgar de 8 a 10 respectivamente, en ambos casos fue ne-

cesario practicarle medidas especiales de recuperación con resultados satisfactorios. El tercer niño, nació con Apgar , 2 al ler. minuto, siguió con 4 al 5\% e igual puntaje al 10\% a pesar de los cuidados intensivos administrados; fué colocado en incubadora y falleció a las 20 horas de nacido, comprobándose a la autopsia la presencia de una grave malformación cardíaca.

\section{VI - Resumen y Conclusiones}

Se estudiaron 30 pacientes con embarazo a término, en trabajo de parto espontáneo o inducido, durante el cual se había diagnosticado la presencia de sufrimiento fetal agudo, por métodos clínicos, basados en las modificaciones de la F.C.F., a saber: A) Taquicardia, B) Taquicardia $y / 0$ Dips Tipo II y C) Bradicardia; a las cuales se administró Orciprenalina (Alupent) en infusión I.V. continua y a dosis variable entre 10 y $20 \mathrm{mi}-$ crogramos por minuto, registrando clínicamente los efectos de la droga sobre las constantes materno fetales y relacionando diversos parámetros con el estado del recién nacido, evaluado por el índice de Apgar al 1\% y 5 : minutos.
Los resultados obtenidos pueden resumirse en la siguiente forma:

1) De treinta (30) pacientes estudiados, solo seis (6) recién nacidos estaban deprimidos al ler. minuto; habiéndose recuperado satisfactoriamente tres (3) de ellos, pasando así al grupo vigoroso al 5 ? minuto.

2) De los seis (6) recién nacidos deprimidos al ler. minuto, en dos (2) se administró la droga por menos de veinte (20) minutos y en otros dos (2) por un período mayor de 60 minutos, lo que nos permite concluir que el Alupent debe ser aplicado por un tiempo mayor de $20 \mathrm{mi}$ nutos, dando así oportunidad al feto de recobrar una homeostasis normal $y$ por consiguiente nacer con un alto puntaje de Apgar.

3) No se encontró diferencia significativa entre los recién nacidos por vía vaginal y por cesárea con relación al Apgar al 1: y 5\% minutos; sinembargo consideramos que el pronóstico del recién nacido depende en gran medida del momento y la forma de decidir el parto (vaginal o cesárea ).

4) Los efectos sobre las constantes materno-fetales son similares a los observados por otros investigadores, haciendo notar que la Orciprenalina (Alupent) a las dosis administradas ( 10 a 20 microgramos por minuto) produjo en todos los casos inhibición de la contractilidad uterina, sin producir efecto mayor sobre la tensión arterial materna, aunque sí taquicardia.

Igualmente en el feto se pudo observar elevación de la F.C.F., pero este efecto fue mucho menor y de desarrollo progresivamente lento con relación a la F.C.M. 
5) Los recién nacidos que ofrecieron mayor dificultad a la recuperación intrauterina fueron los del grupo -C- (Bradicardia permanente).

6) Por los estudios realizados por otros investigadores y por los resultados del presente trabajo, podemos afirmar que la Orciprenalina (Alupent) administrada a la dosis recomendada suprime un factor causal del sufrimiento fetal en forma rápida, segura e inocua para el feto y la madre; favoreciendo así la recuperación intrauterina y permitiendo obtener un recién nacido en óptimo estado de vitalidad.

\section{Summary and Conclusions}

Thirty patients with at term pregnancy were studied during spontaneous or induced labor, during which period the presence of acute fetal suffering has been diagnosed through clinical methods, based on the F.C.F. modifications, to with: (A) Tachycardia; (B) Tachycardia and/or Type II Dips; and (C) Bradycardia. In all cases Orciprenaline (Alupent) was given through I.V. continuous infusion at variable doses fluctuating from 10 to 20 micrograms per minute; the effects of the drug were clinic ally recorded on the maternal fetus constants and various parameters were related with the status of the newly born, evaluated through the Apgar index at the 1 st. and 5 th. minute.

\section{BIBLIOGRAFIA}

1 ALTHABE, O., SCHWAREZ, A., POSE, SV., ESCARCENA, L. and CALDEYRO BARCIA, R. Effects on fetal heart rate p02 of oxygen Administration to the mother. Am. J. Obst. Gynec. 98: 858-870, 1967.

2 ARTO-MEDRANO, F. CASTAÑO, J. L., VERGES, A. Posibilidades terapéuticas de la Orciprenalina en Obstetricia. "El feto de riesgo elevado". Pág. 325 la. ed. 1971.
3 BERENDES, HEINS W. Sufrimiento fetal: Su significación en el desarrollo neurológico y mental infantil. "Factores perinatales que afectan el desarrollo humano". Pág. 222, 1972.

4 CALDEYRO BARCIA, R. Sufrimiento Fetal Intraparto. Fisiopatología. V Congreso Urug. Ginecotocol. Montevideo, 1969. Págs. 529-559.

5 CALDEYRO BARCIA, R. MENDEZ BAUER, C. POSEIRO, J. J. ESCARCEMA, L. A., POSE, S. V., ARNT, IC., GULIN, L., ALTHABE, D. Y BIENIAEZ, J. Bases para el diagnóstico pre$\mathrm{coz}$ del sufrimiento fetal intraparto. Gac. Med. Mex. 96: 687, 1966.

6 CALDEYRO BARCIA, R., MAGAÑA, J. M. CASTILLO, J. B., POSEIRO, J. J., MENDEZ BAUER, C., POSE, S. V., ESCARCENA L. A., CASACUBERTA, C., BUSTOS J. Y GOUSI G. Nuevo enfoque para el tratamiento del sufrimiento fetal agudo intraparto. "Factores perinatales que afectan el desarrollo humano". Pág. 241. 1972.

7 ESTEBAN ALTIRRIBA, J. y otros autores. "Sufrimiento fetal en el parto". 1a. Ed. 1967.

8 FONSECA, D. El sufrimiento fetal y sus consecuencias en el recién nacido $y$ en el niño. V Congr. Urug. Gineco-tocol. Montevideo; 1969

9 FUSTER, R. El interés por el sufrimiento del feto. Progresos de Obst. y Ginec. 9: $277 ; 1966$.

10 MAgAña, J. M., GueVARA, RUBio, G. POSEIRO, J. J. Y CALDEYRO BARCIA, R. ACción de la Orciprenalina (Alupent) sobre la contractilidad uterina del útero humano grávido, el sistema cardiovascular materno y la frecuencia cardíaca fetal. $V$ Congres. Urug. Ginetocol. Montevideo, 1969. Piágs. 615-630.

11 MENDEZ BAUER, C. MONLEON, J. GUEVARA RUBIO, G. BERMEO, L. CARRILLO ANDRADE, A. YABO R. y CALDEYRO BARCIA R. Test clínico para valorar el estado fetal durante el parto. $V$ Cong. Urug. Ginetocol. Montevideo 1969. Pág. 595.

12 MONLEON ALEGRE, F. J. Tratamiento con Orciprenalina del feto de alto riesgo. El feto de riesgo elevado. 1a. Ed. 1971. Pág. 339.

13 MONLEON ALEGRE, F. J. Efectos de la administración brusca de Orciprenalina sobre 
el binomio feto materno. "El feto de riesgo elevado". 1a. Ed. 1971 Pág. 283.

14 MENDEZ BAUER, C., MONLEON, J. GUEVARA RUBIO, G., CASACUBERTA G., BUSTOS R., GIUSSI, G., ESCARCENA L., YABO, R. Y CALDEYRO BARCIA, R. Cambios de la F. C. F. asociados a sufrimiento fetal agudo intraparto. "Factores Perinatales que afectan el desarrollo humano". Pág. 174, 1972.

15 POSE, S. V., CASTILLO, J. B., MORA ROJAS, E., SOTO YANCES A. Y CALDEYRO BARCIA, R. Prueba de tolerancia fetal a las contracciones uterinas inducidas. $V$ Cong. Urug. Ginetocol. Montevideo. 1969. Tomo I. Págs. 641-664.

16 POSE, S. V., TEMESIO, P., MARTINO, P. V., SOTO YANCES, A. MORA ROJAS, E. CASTILLO, J. Y CALDEYRO BARCIA R. Evaluación de la vitalidad fetal durante el embarazo. III Jornada Rioplatense de Diabetes. (Buenos Aires). 1968.

17 POSEIRO, J. J. Sufrimiento fetal intraparto. Causas. V Congr. Urug. Ginetocol. Montevideo 1969. Pág. 560-594.
Rev. Col. Obst. y Ginec.

18 SALING E. "El niño desde el punto de vista obstétrico". 1a. Ed. 1969.

19 SOTO YANCES, A. Uso clínico de la Orciprenalina como inhibidor de la contractilidad uterina. VIII Congr. Col. Obst. Ginec. Manizales, 1969.

20 SOTO YANCES, A. "Test clínico de tolerancia fetal". Para publicación.

21 COBO, E., KAFURI, S.: "Inhibición de la contractilidad del útero en la amenaza de parto prematuro mediante el uso de Orciprenalina (Alupent). Presentado en VIII Congr. Col. de Obst. y Gin. Manizales, 12. 69.

\section{AGRADECIMIENTO}

Los autores desean expresar su agradecimiento al personal científico de la Clínica Universitaria Rafael Calvo C. y a los Departamentos de Obstetricia y Ginecología de la Universidad de Cartagena y del Instituto Colombiano de Seguros Sociales, por su invaluable colaboración para la realización de este trabajo. 